\title{
Modelagem e Análise de dados MMT 3D segundo Ambientes Altamente Resistivos em Águas Profundas
}

Frayzer L. de Almeida ${ }^{(1)}$, Carlos A. S. Ferreira ${ }^{(2)}$ \& Rodrigo E. da C. Silva ${ }^{(3)}$

(1) Campus de Castanhal/UFPA, (2) ANP e (3) UEPA

Copyright 2016, SBGf - Sociedade Brasileira de Geofísica

Este texto foi preparado para a apresentação no VII Simpósio Brasileiro de Geofísica, Ouro Preto, 25 a 27 de outubro de 2016. Seu conteúdo foi revisado pelo Comitê Técnico do VII SimBGf, mas não necessariamente representa a opinião da SBGf ou de seus associados. É proibida a reprodução total ou parcial deste material para propósitos comerciais sem prévia autorização da SBG.

\section{ABSTRACT}

This work proposes numerical solutions for the 3D MMT forward problem using finite-elements and two EM potentials. Answers to 1D MMT obtained through semianalytical solutions are used for modeling and calibration of the 3D MMT proposed here. Some limitations of the 1D MMT forward modeling are discussed when compared to the MMT forward modeling of 3D geoelectrical models, as well as the advantages and limitations of the 3D MMT forward modeling according to the TM and TE modes, when interpretation of bidimensional sections of 3D geoelectrical models are considered.

\section{RESUMO}

Este trabalho propõe soluções numéricas para o problema direto do MMT 3D usando o método dos elementos finitos tridimensionais através dos potenciais EM $(\mathbf{A}, \Phi)$. Respostas do MMT 1D, obtidos através de solução semi-analítica, são usadas na modelagem e calibração da solução numérica do MMT 3D aqui proposta. São discutidas limitações da modelagem direta do MMT 1D em relação à modelagem direta do MMT 3D na interpretação de modelos geoelétricos 3D, assim como as vantagens e limitações da modelagem direta do MMT 3D segundo os modos TM e TE sobre a interpretação de seções bidimensionais de modelos geoelétricos 3D.

\section{INTRODUÇÃO}

O método Magnetotelúrico Marinho - MMT é um dos métodos eletromagnéticos - EM com destaque na prospecção de hidrocarbonetos em meio altamente resistivo localizado em águas profundas e ultraprofundas. Usado em conjunto com a sísmica, é de grande auxílio na confirmação da existência de hidrocarbonetos, no mapeamento do contorno de reservatórios, e também contribui na diminuição da ambiguidade na interpretação geológica.

Dessa forma, existe a necessidade de maior interesse de pesquisa sobre o método MMT como, por exemplo, no desenvolvimento de equipamentos de aquisição de sinal eletromagnéticos mais acurados em águas profundas, assim como o desenvolvimento de metodologias computacionais para a simulação de respostas eletromagnéticas de dimensões 1D, 2D e 3D altamente

resistivas como, por exemplo, as respostas eletromagnéticas verificadas nas bacias costeiras brasileiras que são provenientes de extensas e profundas estruturas salinas localizadas em águas profundas.

Com dados MMT 3D (modo TM) simulados à partir de um modelo que representa uma estrutura salina no Golfo do México e usando modelagem inversa 2D, Hoversten, Constable e Morrison (2000), solucionam o problema inverso usando quatro algoritmos distintos, entre os quais estão o algoritmo do gradiente conjugado não linear (Mackie et al., 1997) e o algoritmo de Occam 2D (deGroot-Hedlin and Constable, 1990). Para a solução do problema direto do MMT 3D foi aplicado o método numérico das diferenças finitas (Mackie et al.,1993).

Através de dados MMT reais da Bacia de Santos e usando modelagem inversa $2 \mathrm{D}$ segundo o método do gradiente conjugado não-linear, foi possível Pinto (2009) obter boa resolução do contato sal-sedimento, assim como a sua profundidade aproximada. Para a solução do problema direto do MMT 2D (modo TM e modo TE), foi aplicado o método numérico das diferenças finitas.

Para a modelagem direta de dados do MMT 3D aplicada na prospecção de hidrocarbonetos em ambientes altamente resistivos localizados em águas profundas, este trabalho propõe uma metodologia através do método dos elementos finitos tridimensionais usando os potenciais EM ( $\mathbf{A}, \Phi)$ ), analogamente ao proposto por Almeida e Rijo (2011), o qual usa a referida metodologia, porém para a modelagem direta de dados do mCSEM 3D.

Para fim de calibração e avaliação numérica dos resultados provenientes da modelagem direta do MMT 3D aqui proposta, apresentamos comparações entre as resistividades aparentes e as fases provenientes de modelos geoelétricos unidimensionais com características de ambientes altamente resistivos em águas profundas. As respostas advindas dos modelos unidimensionais são obtidas através da modelagem direta do MMT 1D segundo formulação proposta por Rijo (2002).

Através de respostas do MMT 1D e 3D, segundo modelos geoelétricos altamente resistivos em águas profundas, observamos uma limitação da modelagem do MMT 1D em relação à modelagem do MMT 3D na interpretação de modelos geoelétricos 3D. Em seguida, através de pseudo-seções de resistividades aparentes do MMT 3D é observado o melhor delineamento do ambiente altamente resistivo em águas profundas segundo o modo TM em relação ao modo TE na direção do comprimento do corpo resistivo de tal seção. Assim como, através de pseudo-seções de resistividades aparentes do MMT 3D é observado o melhor 
delineamento do ambiente altamente resistivo em águas profundas segundo o modo TE em relação ao modo TM na direção da largura do corpo resistivo da referida seção. Finalmente, através destas pseudoseções (modos TM e TE) observamos que o MMT não é capaz de diferenciar o corpo reservatório com hidrocarbonetos do corpo salino.

\section{O PROBLEMA DIRETO DO MMT 3D SEGUNDO OS POTENCIAIS EM $(\mathbf{A}, \Phi)$}

Através das equações de Maxwell no $\mathrm{SI}$, em um meio não magnético, no domínio da frequência e em termos dos campos secundários elétrico $\mathbf{E}^{s}$ e magnético $\mathbf{H}^{s}$, temos:

$$
\begin{gathered}
\nabla \cdot\left(\varepsilon_{0} \mathbf{E}^{s}\right)=0, \\
\nabla \times \mathbf{H}^{s}-\left(\sigma^{p}+\Delta \sigma\right) \mathbf{E}^{s}=\mathbf{J}^{s}, \\
\nabla \cdot\left(\mu_{0} \mathbf{H}^{s}\right)=0 \\
\nabla \times \mathbf{E}^{s}+i \omega \mu_{0} \mathbf{H}^{s}=\mathbf{0},
\end{gathered}
$$

em que $\varepsilon_{0}=8,854 \cdot 10^{-12} \quad F / m$ e $\mu_{0}=4 \pi \cdot 10^{-7}$ $H / m$ são a permissividade elétrica no vácuo e a permeabilidade magnética no vácuo, respectivamente. $\sigma^{p}$ e $\Delta \sigma$ são a condutividade elétrica do meio homogêneo e a diferença de condutividade entre o meio homogêneo e a heterogeneidade, respectivamente. $\mathbf{J}^{s}=\Delta \sigma \mathbf{E}{ }^{s}$ é a densidade de correntes secundárias e $\omega=2 \pi f$ a freqüência angular, enquanto $f$ a freqüência linear. Em virtude das Eqs.(3) e (4) podemos expressar os campos $\mathbf{E}^{s}$ e $\mathbf{H}^{s}$ em termos dos potenciais secundários magnético $\mathbf{A}^{s}$ e elétrico $\Phi^{s}=i \omega \mu_{0} \phi^{s}$ da seguinte forma

$$
\begin{gathered}
\mathbf{H}^{s}=\nabla \times \mathbf{A}^{s}, \\
\mathbf{E}^{s}=-i \omega \mu{ }_{0} \mathbf{A}^{s}-\nabla \Phi^{s} .
\end{gathered}
$$

Das Eqs.(5) e (6), a equação Eq.(2) torna-se a equação rotacional do rotacional

$$
\nabla \times \nabla \times \mathbf{A}^{s}+i \omega \mu_{0} \sigma \mathbf{A}^{s}+\sigma \nabla \Phi^{s}=\mathbf{J}^{s} .
$$

A Eq.(7), quando expressa segundo o método dos elementos finitos, resulta em uma matriz assimétrica que pode apresentar modos espúrios e instáveis numericamente (Souza, 2007). Para evitar esta dificuldade, aplica-se a identidade vetorial:

$$
\nabla \times \nabla \times \mathbf{A}=-\nabla^{2} \mathbf{A}+\nabla(\nabla \cdot \mathbf{A}),
$$

juntamente com a incorporação do calibre de Coulomb:

$$
\nabla \cdot \mathbf{A}^{s}=0 \text {, }
$$

dando origem à equação:

$$
\nabla^{2} \mathbf{A}^{s}-i \omega \mu_{0} \sigma\left(\mathbf{A}^{s}+\nabla \phi^{s}\right)=-\mathbf{J}^{s},
$$

para a qual a sua forma discretizada é estável numericamente (Souza, 2007). Na derivação da Eq.(7) definiu-se o potencial escalar reduzido $\Phi^{s}=i \omega \mu_{0} \phi^{s}$, o que resulta em uma matriz de elementos finitos simétrica, quando a equação resultante da derivação está expressa na sua forma discretizada segundo os elementos finitos tridimensionais. Tomando o divergente da Eq.(7) e considerando a propriedade $\nabla \cdot \nabla \times \mathbf{H}=0$, tem-se:

$$
i \omega \mu_{0} \nabla \cdot\left(\sigma \mathbf{A}^{s}\right)+i \omega \mu_{0} \nabla \cdot\left(\sigma \nabla \phi^{s}\right)=\nabla \cdot \mathbf{J}^{s} .
$$

Desta forma as Eqs.(8) e (9) são solucionadas simultaneamente através do método dos elementos finitos tridimensionais (Souza, 2007) aplicando o critério de Galerkin (Becker; Carey; Oden, 1981), do qual se obtém um sistema linear associado a uma matriz complexa, simétrica e esparsa. Este sistema é resolvido numericamente pelo método Gauss-Seidel (Barroso et al., 1987), resultando assim nos potenciais $\mathbf{A}^{s}$ e $\phi^{s}$.

Na formulação do problema direto tridimensional através os potenciais secundários, os campos elétricos e magnéticos primários ( $\mathbf{E}^{p}, \mathbf{H}^{p}$ ) são obtidos através da modelagem direta do MMT 1D segundo formulação proposta por Rijo (2002).

A metodologia que este trabalho propõe é idêntica à metodologia proposta por Almeida (Almeida, 2011), uma vez que ambas aplicam o critério de Galerkin (Becker; Carey; Oden, 1981) sobre as Eqs.(8) e (9), juntamente com a aplicação da identidade

$$
-\int_{\Omega_{e}} N_{k} \frac{\partial N_{l}}{\partial x} d \Omega_{e}=\int_{\Omega_{e}} N_{l} \frac{\partial N_{k}}{\partial x} d \Omega_{e}-\oint_{\partial \Omega_{e}} N_{k} N_{l} d \partial \Omega_{e},
$$

tanto à esquerda quanto à direta da Eq.(9), porém Almeida (2011) propõe esta metodologia na modelagem numérica tridimensional do mCSEM, diferentemente deste artigo que à propõe na modelagem numérica tridimensional, mas para o MMT. Ao se aplicar a identidade da Eq.(10) à direita da Eq.(9), estamos admitindo a continuidade da divergência de $\mathbf{J}^{s}$ (Almeida, 2011).

A Eq.(10) é uma identidade das funções bases $\left(N_{k}\right.$ e $N_{l}$ ) dos elementos finitos tridimensionais (nas direções y e $z$, a expressão é semelhante, utilizando derivadas parciais nas direções $y \quad e \quad z$, respectivamente) (Zienkiewicz and Taylor, 2000).

De posse dos campos eletromagnéticos secundários ( $\mathbf{E}^{s}, \mathbf{H}^{s}$ ) e dos campos eletromagnéticos primários ( $\mathbf{E}^{p}, \mathbf{H}^{p}$ ), determinamos os campos eletromagnéticos total do MMT 3D. Finalmente, através de duas polarizações distintas dos campos eletromagnéticos total do MMT 3D, ou seja, $\left(\mathbf{E}^{1}, \mathbf{H}^{1}\right)$ e $\left(\mathbf{E}^{2}, \mathbf{H}^{2}\right)$ e para uma mesma frequência, é possível calcular as resistividades aparentes $\rho_{x y}$ e $\rho_{y x}$ que são definidas por:

$$
\begin{aligned}
& \rho_{T M}=\rho_{x y}=\frac{1}{\omega \mu_{0}}\left|Z_{x y}\right|^{2} \\
& \rho_{T E}=\rho_{y x}=\frac{1}{\omega \mu_{0}}\left|Z_{y x}\right|^{2} .
\end{aligned}
$$

e

Em que as componentes do tensor de impedância $Z_{x y}$ e $Z_{y x}$ são expressas por:

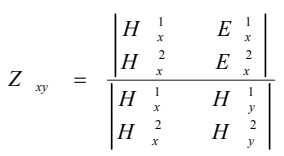




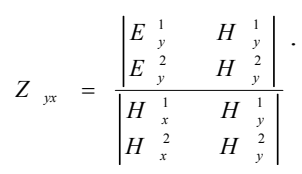

De modo que o cálculo dessas componentes é feito através do tensor de impedância:

$$
\left[\begin{array}{l}
E_{x} \\
E_{y}
\end{array}\right]=\left[\begin{array}{ll}
Z_{x x} & Z_{x y} \\
Z_{y x} & Z_{y y}
\end{array}\right]\left[\begin{array}{l}
H_{x} \\
H_{y}
\end{array}\right],
$$

usando-se as duas polarizações distintas, o que resulta nos seguintes sistemas lineares complexos:

$$
\begin{aligned}
& E_{y}^{1}=Z_{y x} H_{x}^{1}+Z_{y y} H_{y}^{1} \\
& E_{y}^{2}=Z_{y x} H_{x}^{2}+Z_{y y} H_{y}^{2}
\end{aligned}
$$

e

$$
\begin{aligned}
& E_{x}^{1}=Z_{x x} H_{x}^{1}+Z_{x y} H_{y}^{1} \\
& E_{x}^{2}=Z_{x x} H_{x}^{2}+Z_{x y} H_{y}^{2}
\end{aligned}
$$

Dessa forma, a resistividade aparente $\rho_{x y}$ é caracterizada segundo o seu modo de propagação eletromagnética como o modo transversal magnético com relação à direção $z$ ou simplesmente modo TM. Da mesma maneira, a resistividade aparente $\rho_{y x}$ é caracterizada segundo o seu modo de propagação transversal elétrico com relação à direção $z$ ou simplesmente modo TE.

\section{EXEMPLOS DE AMBIENETES ALTAMENTE RESISTIVOS 1D e 3D EM ÁGUAS PROFUNDAS}

O modelo geoelétrico MMT 1D segundo características altamente resistivas em águas profundas aqui proposto, (Fig.1), é formado pela camada de ar com resistividade elétrica $\rho_{0}$ infinita. A camada do mar, com resistividade $\rho_{1}$ igual a $0,33 \mathrm{ohm}-\mathrm{m}$ e espessura $h_{1}$ igual a $2000 \mathrm{~m}$. A camada das rochas sedimentares encaixantes (folhelho, siltito, etc), com resistividade $\rho_{2}$ igual a 1,0 ohm-m e espessura $h_{2}$ igual a $1000 \mathrm{~m}$. A camada de sal (rochas sedimentares salinocarbonáticas) com resistividade $\rho_{3}$ igual a 100 ohm-m e espessura $h_{3}$ igual a $2000 \mathrm{~m}$. Em seguida a camada plano paralela, a qual representa o reservatório de hidrocarbonetos, possui resistividade $\rho_{4}$ igual a 50 ohm-m e uma espessura igual a 1500 metros. Finalmente a camada das rochas sedimentares encaixantes apresenta resistividade $\rho_{5}$ igual a 1,0 ohm$\mathrm{m}$.

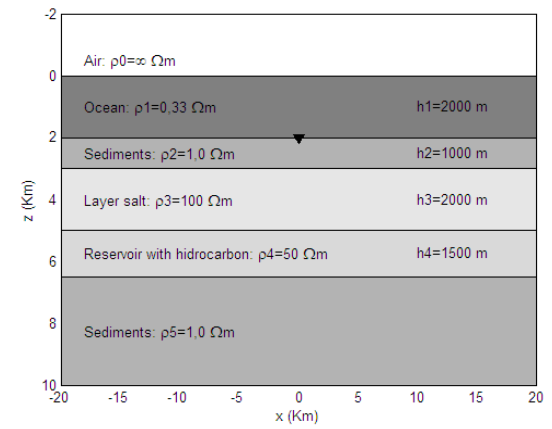

Figura 1 - Modelo geoelétrico 1D com camada de sal e reservatório com hidrocarbonetos.

Os campos eletromagnéticos, MMT 1D, referentes ao modelos geoelétricos unidimensionais acima descritos, é solucionado analiticamente a partir das equações de Maxwell no SI, em um meio não magnético e no domínio da frequência segundo formulação proposta por Rijo (2002).

O modelo geoelétrico MMT 3D segundo características altamente resistivas em águas profundas, aqui proposto, Figs.2 e 3, é expresso pelas seções segundo as perspectivas do plano $z \times x$ e $z \times y$, respectivamente.

Em que, para o plano $z \times x$, a camada de sal apresenta um comprimento na direção $x$ de $60 \mathrm{~km}$, uma espessura $h_{3}$ igual a $2000 \mathrm{~m}$ e uma resistividade elétrica $\rho_{3}$ igual a 100 ohm-m. A camada que representa o reservatório de hidrocarbonetos possui um comprimento na direção $x$ de $30 \mathrm{~km}$, uma espessura $h_{4}$ igual $1500 \mathrm{~m}$, está a uma profundidade do assoalho marinho de $3000 \mathrm{~m}$ e apresenta uma resistividade $\rho_{4}$ igual a 50 ohm-m (Fig.2).

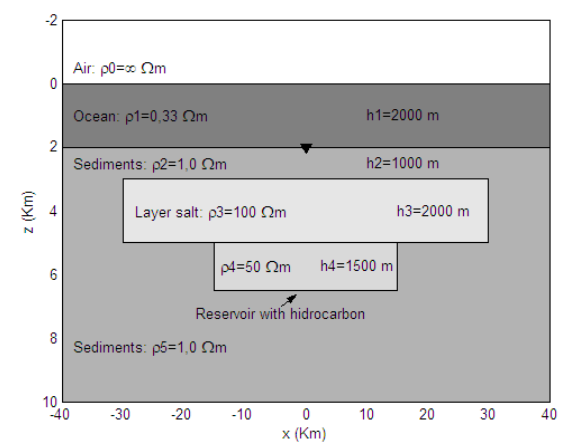

Figura 2 - Seção no plano $z \times x$ referente ao modelo geoelétrico 3D com camada de sal e reservatório com hidrocarbonetos.

Já para o plano $z \times y$, a camada de sal apresenta uma largura na direção $y$ de $40 \mathrm{~km}$ e a camada que representa o reservatório com hidrocarbonetos possui uma largura na direção $y$ de $20 \mathrm{~km}$ (Fig.3). 


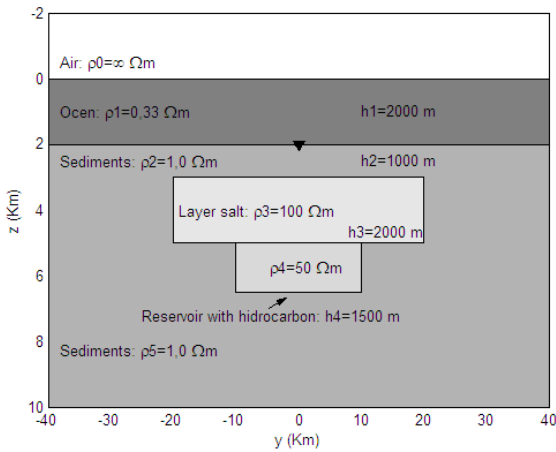

Figura 3 - Seção no plano $z \times y$ referente ao modelo geoelétrico 3D com camada de sal e reservatório com hidrocarbonetos.

\section{A CALIBRAÇÃO DAS RESPOSTAS DO MMT 3D EM RELAÇÃO AS RESPOSTAS DO MMT 1D}

Para verificarmos a calibração das soluções devido à modelagem direta do MMT 3D (modos TM e TE), usamos o modelo geoelétrico 3D caracterizado pelas Figs 2 e 3 . Desta forma, temos o modelo geoelétrico acima descrito e modelado segundo a metodologia do MMT 3D apresentada na seção anterior a qual denominamos de Experimento 1-3D (modos TM e TE). Para o modelo acima, um receptor está afixado no assoalho marinho, na coordenada $x=0$ e $y=0$, operando em 14 períodos distintos entre $10^{0}$ e $10^{4}$ segundos distribuídos uniformemente em escala logarítmica.

Assim comparamos as respostas do MMT 3D (Modos TM e TE) em relação à resposta através da modelagem direta do MMT 1D (Rijo, 2002) segundo o modelo geoelétrico 1D apresentado na Fig. 1. Esta modelagem direta 1D versus o modelo geoelétrico 1D (Fig.1) corresponde ao que denominamos de Experimento 1-1D.

São apresentadas nas Figs. 4 e 5 as resistividades aparentes em ohm-m e as fases em graus, respectivamente, referentes ao modelo geoelétricos do MMT 3D (modos TM e TE): Experimentos 1-3D e o modelo geoelétrico do MMT 1D: Experimento 1-1D.

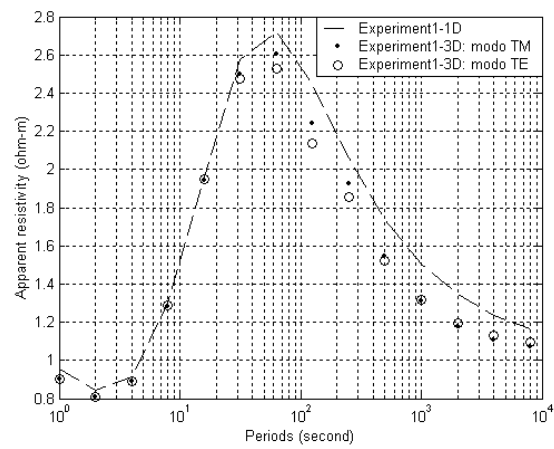

Figura 4 - Resistividades aparentes a partir dos modelos geoelétricos do MMT 3D (modos TM e TE) (Figs 2 e 3) e 1D (Fig 1).

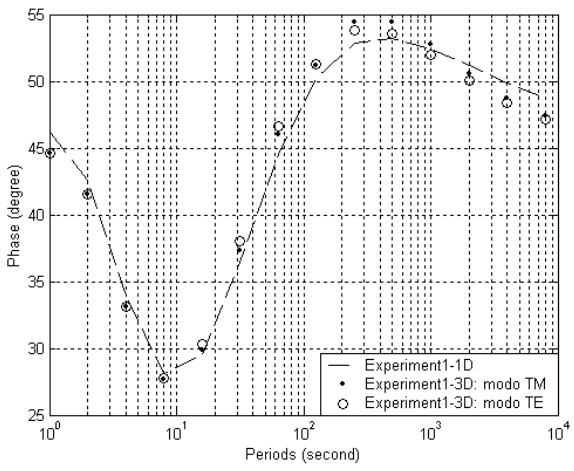

Figura 5 - Fases a partir dos modelos geoelétricos do MMT 3D (modos TM e TE) (Figs 2 e 3) e 1D (Fig 1).

Devido aos receptores estarem afixados no assoalho marinho, na coordenada $x=0$ e $y=0$, e das grandes dimensões (comprimento e largura) da camada de sal e da camada com hidrocarbonetos referente ao modelo geoelétrico 3D (Figs. 2 e 3), observa-se tanto na Figura 4 quanto na Fig. 5 a aproximação das resistividades aparentes (Experimento 1-3D: modos TM e TE) e das fases (Experimento 1-3D: modos TM e TE), em relação às resistividades aparentes e fases (Experimento1-1D), respectivamente, referentes ao modelo geoelétrico 1D (Fig.1). Dessa forma, as respostas 3D (modos TM e TE), para coordenada em questão, apresentam forma e aproximação coerentes em relação à resposta 1D.

\section{RESULTADOS DO MMT 3D SEGUNDO AMBIENTES ALTAMENTE RESISTIVOS EM ÀGUAS PROFUNDAS}

Finalmente vamos analisar o comportamento das pseudo-seções de resistividades aparentes segundo a modelagem do MMT 3D (modos TM e TE) através do modelo geoelétrico 3D representado pelas Figs.2 e 3.

Para uma primeira etapa de análise dos dados do MMT 3D através do modelo acima (Figs.2 e 3), os receptores estão afixados no assoalho marinho sobre o eixo $\mathrm{x}$, com espaçamento de 2500 em 2500 metros, totalizando 33 pontos de sondagens do MMT, cobrindo uma extensão de $80 \mathrm{~km}$ e operando em 14 períodos distintos entre $10^{\circ}$ e $10^{4}$ segundos e distribuídos uniformemente em escala logarítmica. Dessa forma, são apresentadas nas Figs. 6 e 7 as pseudo-seções de resistividades aparentes em ohm$\mathrm{m}$, segundo os modos TM e TE, respectivamente, referentes ao modelo geoelétrico do MMT 3D (Figs.2 e $3)$. Estas pseudo-seções apresentam os períodos em escala logarítmica na base dez, e estão associadas à seção no plano $z \times x$ apresentada na Fig.2. 


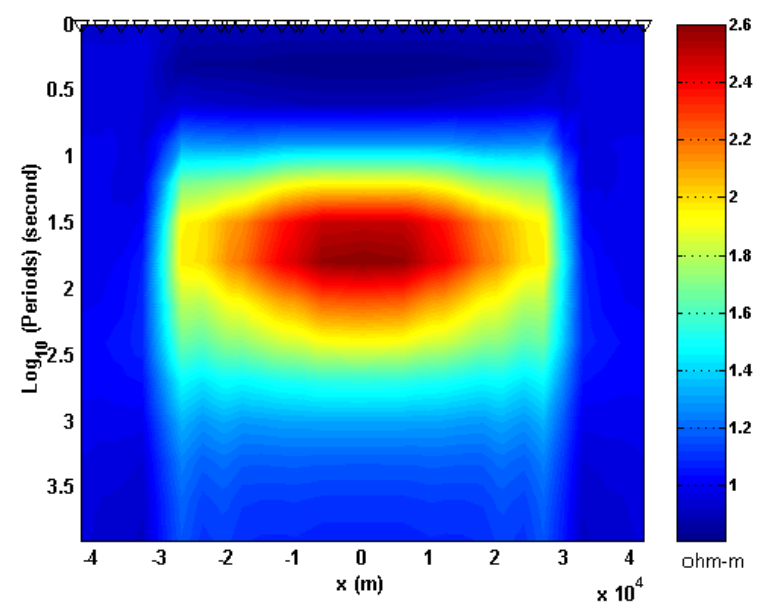

Figura 6 - Pseudo-seção de resistividades aparentes do MMT 3D segundo o modo TM, associada à seção no plano $z \times x$ apresentada na Fig.2.

Comparando os resultados das Figs. 6 e 7, observa-se um delineamento mais aproximado das resistividades aparentes segundo o modo TM (Fig.6) em relação ao modo TE (Fig.7), quando associados à geometria do ambiente resistivo observado na seção no plano $z \times x$ (Fig.2) do modelo geoelétrico 3D (Figs.2 e 3).

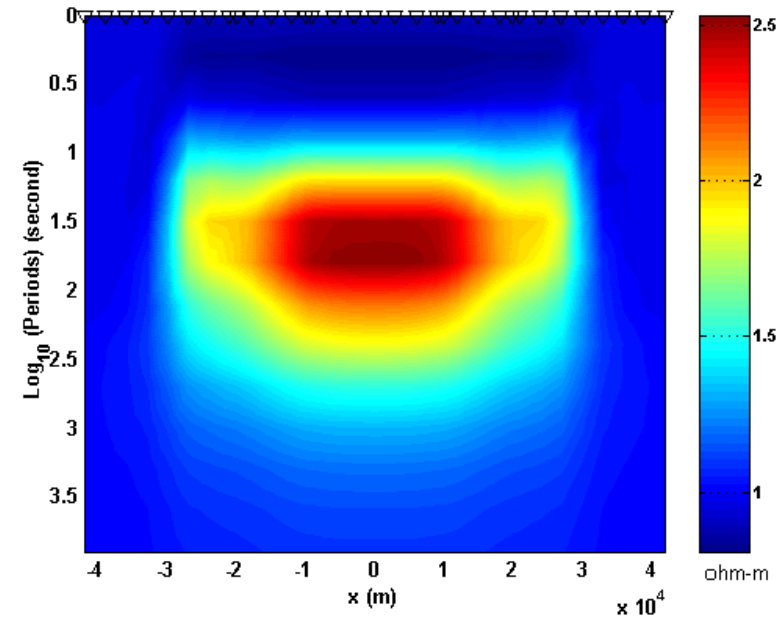

Figura 7 - Pseudo-seção de resistividades aparentes do MMT 3D segundo o modo TE, também associada à seção no plano $z \times x$ apresentada na Fig.2.

Agora, para a segunda e última etapa de análise dos dados do MMT 3D através do modelo geoelétrico acima (Figs.2 e 3), os receptores estão também afixados no assoalho marinho, mas agora sobre o eixo y, com espaçamento de 2500 em 2500 metros, totalizando também 33 pontos de sondagens do MMT, cobrindo uma extensão de $80 \mathrm{~km}$ e operando em 14 períodos distintos entre $10^{\circ}$ e $10^{4}$ segundos distribuídos uniformemente em escala logarítmica. Assim, são apresentadas nas Figs. 8 e 9 as pseudo-seções de resistividades aparentes em ohm-m, segundo os modos TM e TE, respectivamente, referentes ao modelo geoelétrico do MMT 3D caracterizado pelas Figs. 2 e 3 . Estas pseudo-seções também apresentam os períodos em escala logarítmica na base dez, e estão associadas à seção no plano $z \times y$ apresentada na Fig. 3 .

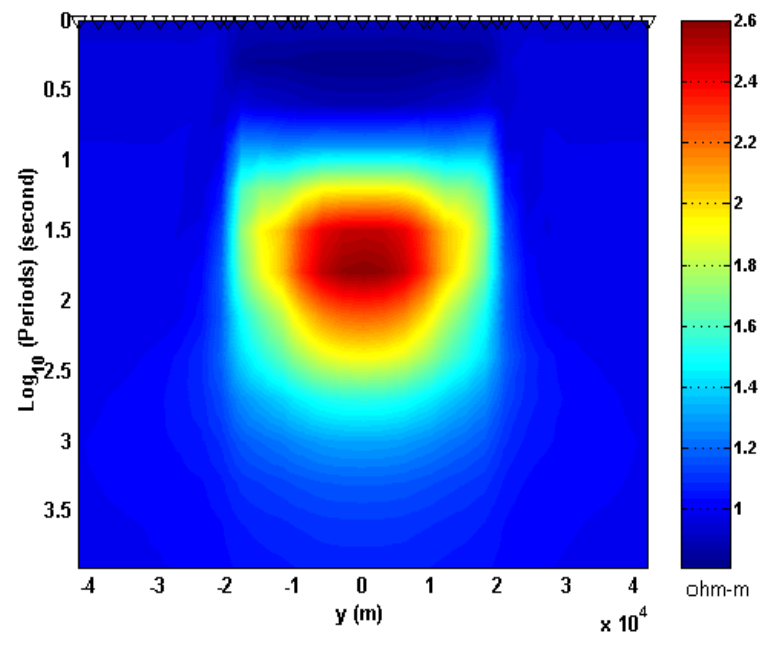

Figura 8 - Pseudo-seção de resistividades aparentes do MMT $3 \mathrm{D}$ segundo o modo TM, associada à seção no plano $z \times y$ apresentada na Fig.3.

Comparando os resultados das Figs. 8 e 9, observa-se um delineamento mais aproximado das resistividades aparentes segundo o modo TE (Fig.9) em relação ao modo TM (Fig.8), quando associados à geometria do ambiente resistivo observado na seção no plano $z \times y$

(Fig.3) do modelo geoelétrico 3D (Figs.2 e 3).

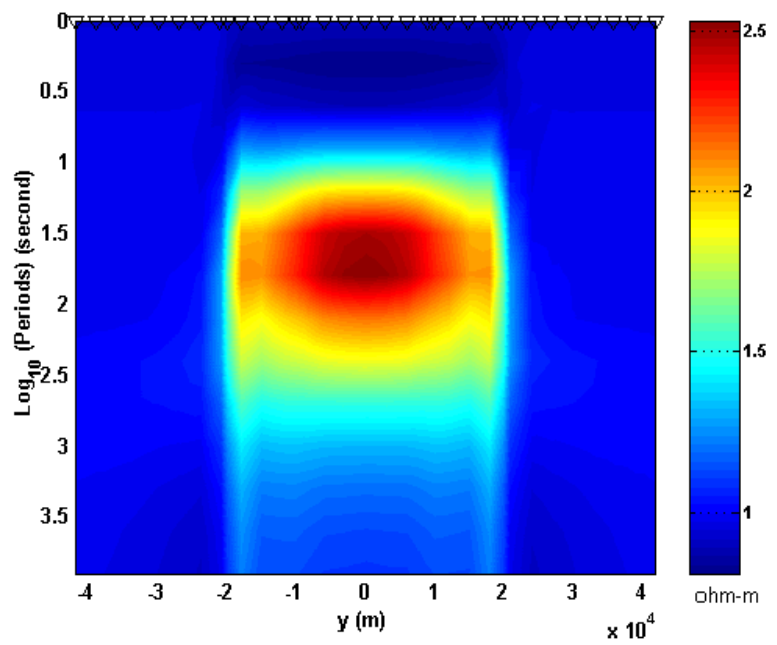

Figura 9 - Pseudo-seção de resistividades aparentes do MMT $3 \mathrm{D}$ segundo o modo TE, também associada à seção no plano $z \times y$ apresentada na Fig.3.

Observamos nas Figs. 6-9 que o MMT 3D tem resposta com contraste necessário para a diferenciação, quando caracterizado, de forma simplificada, por ambientes altamente resistivos em águas profundas (Fig.2 e 3). Pois 
é possível observar o delineamento lateral do corpo resistivo (corpo salino e corpo reservatório com hidrocarbonetos), assim como o delineamento do seu topo e da sua base através das resistividades aparentes, mostrando assim coerência com o modelo geoelétrico 3D (Fig.2 e 3), uma vez que este de fato apresenta variação tanto lateral quanto vertical de resistividade elétrica na região de interesse. Diferentemente de respostas do MMT 1D que não apresentam variação lateral de resistividade elétrica. Dessa forma, é evidenciada uma limitação da modelagem direta do MMT 1D ao se aplicar a modelos geoelétricos unidimensionais voltados para a interpretação de modelos geoelétricos tridimensionais de ambientes altamente resistivos localizados em águas profundas.

Em nenhuma das pseudo-seções de resistividades aparentes, Figs. 6-9, observamos o delineamento do corpo reservatório de hidrocarbonetos, assim como o contato entre o corpo salino e o corpo reservatório com hidrocarbonetos.

\section{CONCLUSÕES}

Inicialmente evidenciamos nas pseudo-seções de resistividades aparentes do MMT 3D (modos TM e TE) a variação lateral de resistividades aparentes (Figs.6-9), diferentemente de repostas do MMT 1D que não apresentam variação lateral de resistividades aparentes.

Observamos nos resultados do MMT 3D (Figs.6 e 7) que as medidas de resistividades aparentes segundo o modo TM, melhor delineiam a geometria do corpo resistivo, em relação às medidas de resistividades aparentes segundo o modo TE. Este melhor delineamento do corpo tridimensional na seção bidimensional (Fig.2) através do MMT 3D (modo TM) é devido à contribuição do campo elétrico deste modo, uma vez que o mesmo é normal à direção da largura do corpo resistivo (de extensão em $y$ de $40 \mathrm{~km}$ ), possibilitando registrar um melhor contraste entre o meio mais resistivo e o meio menos resistivo na direção do comprimento do corpo resistivo (Fig.2).

Também observamos nos resultados do MMT 3D (Figs.8 e 9) que as medidas de resistividades aparentes segundo o modo TE, melhor delineiam a geometria do corpo resistivo, em relação às medidas de resistividades aparentes segundo o modo TM. Este melhor delineamento do corpo tridimensional na seção bidimensional (Fig.3) através do MMT 3D (modo TE) é devido à contribuição do campo elétrico deste modo, uma vez que o mesmo é normal à direção do comprimento do corpo resistivo (de extensão em $x$ de $60 \mathrm{~km}$ ), possibilitando registrar um melhor contraste entre o meio mais resistivo e o meio menos resistivo na direção da largura do corpo resistivo (Fig.3).

Observamos ainda nos resultados 3D (Figs.6-9) que as medidas de resistividades aparentes, tanto segundo o modo TM quanto o modo TE, não diferenciam e/ou identificam o contato entre a corpo salino e o corpo reservatório com hidrocarbonetos, uma vez que não existe uma "grande" e/ou "significativa" razão de resistividade elétrica entre ambos os corpos, evidenciando dessa forma uma limitação do método MMT quando aplicado a ambientes altamente resistivos localizados em águas profundas.

\section{AGRADECIMENTOS}

O autor (F.L.A.) agradece aos Conselhos da Faculdade de Matemática e do Campus de Castanhal/UFPA pela aprovação do projeto de pesquisa que viabilizou o desenvolvimento deste trabalho.

\section{REFERÊNCIAS}

ALMEIDA, F.L. e RIJO, L.-2011-Modelagens Direta e Inversa de Dados mCSEM 3D. RBGf. v.29(2), p. 289-308.

BARROSO, L. C., BARROSO, M. M. A., FILHO, FREDERICO, F. C., CARVALHO, M. L. B., MAIA, M. L.. Cálculo Numérico. Editora Harbra Ltda. São Paulo, 1987.

BECKER, E. B., CAREY, G. F., ODEN, J. T. -1981- Finit elements - An introduction. New Jersey: Prentice-Hall, $258 p$.

deGroot-Hedlin, C., and CONSTABLE, S. C.-1990Occam's inversion to generate smooth, two-dimensional models from magnetotelluric data: Geophysics, n. 55, p. 1613-1624.

HOVERSTEN, G. M., CONSTABLE, S. C., MORRISON, H. F.-2000- Marine magnetotellurics for base-of-salt mapping: Gulf of Mexico field test at the Gemini structure. Geophysics, n. 5, p. 1476-1488.

MACKIE, R. L., LIVELYBROOKS, D. W., MADDEN, T. R., and LARSEN, J. C.-1997- A magnetotelluric investigation of the San Andreas fault at Carrizo Plain, California: Geophys. Res. Lett., 24,1847-1850.

MACKIE, R. L., MADDEN, T. R., and WANNAMAKER, P. W.-1993- Three-dimensional magnetotelluric modeling using difference equations-Theory and solutions: Geophysics,n. 58, p. 215-226.

PINTO, V. R.-2009- O Método Magnetotelúrico Marinho (MMT) na Exploração de Hidrocarbonetos. Dissertação de Mestrado, ON, $63 \mathrm{p}$.

RIJO, L.-2002- Teoria dos Métodos Eletromagnético I, II e III - Notas de aula. Departamento de Geofísica, UFPa.

SOUZA, V. C. T.-2007- Modelagem numérica de dados MCSEM 3D usando computação paralela. Tese de Doutorado, UFPA, $110 \mathrm{p}$.

ZIENKIEWICZ, O. C., TAYLOR, R. L.-2000- The finite element method - Volume 1: the basis. Oxford: Butterworth Heinemann, 2000. 